\title{
Time series analysis of V511 Lyrae photometry
}

\author{
J. Lyytinen ${ }^{1}$, P. Johansson ${ }^{1}$, L. Jetsu ${ }^{1}$, E. Esko ${ }^{1}$, T. Hackman ${ }^{1}$, D. S. Hall ${ }^{2}$, G. W. Henry ${ }^{3}$, S. Kontinen ${ }^{1}$, \\ P. Könönen ${ }^{1,4}$, S. Maisala ${ }^{1}$, A. Palviainen ${ }^{1}$, and K. Ryynänen ${ }^{1}$ \\ 1 Observatory, PO Box 14, 00014 University of Helsinki, Finland \\ 2 Dyer Observatory, Vanderbilt University, Nashville, TN 37235, USA \\ 3 Center of Excellence in Information Systems, Tennessee State University, 330 10th Ave. North, Nashville, \\ TN 37203-3401, USA \\ 4 Helsinki University of Technology, Metsähovi Radio Research Station, Metsähovintie, 02540 Kylmälä, Finland
}

Received 25 August 2000 / Accepted 26 November 2001

\begin{abstract}
Our time series analysis of sixteen $B V$ light curves of the chromospherically active binary V511 Lyr confirmed the 2.7 rotation period uniquely. The seasonal periodicity changes of $3.8 \%$ indicated the presence of detectable surface differential rotation. The significant 2.67455 periodicity in the light curve minimum epochs suggested that the strongest starspot formation in V511 Lyr was concentrated on one stable active longitude.
\end{abstract}

Key words. stars: individual: V511 Lyr (HDE 337518) - stars: variable: general - stars: activity - techniques: photometric

\section{Introduction}

Bidelman (1983) noticed the chromospheric CaII K emission of V511 Lyr (HDE 337518). Due to its strong coronal emission, V511 Lyr appears in both catalogues of the bright extreme ultraviolet sources discovered by the ROSAT-satellite (Pounds et al. 1993; Pye et al. 1995). It has been identified as the optical counterpart of the ROSAT source 2RE 190624+274308 (Mason et al. 1995) and the EUVE source J 1906+274 (Lampton et al. 1997). The spectroscopy by Mulliss \& Bopp (1994) revealed that V511 Lyr is a double-lined binary with chromospheric $\mathrm{H} \alpha$ and CaII $8542 \AA$ emission. Jeffries et al. (1995) determined the orbital period $P_{\text {orb }}=2.73 \pm 0.03$ and concluded that this system probably consists of two mid-K main sequence stars with nearly circular orbits. The photometric rotation period, $P_{\text {phot }}=2.693 \pm 0$ d 005 , obtained by Henry et al. (1995) indicated synchronous rotation. They estimated the spectral-types of the primary $(\mathrm{KOV})$ and the secondary $(\mathrm{K} 3 \mathrm{~V})$, and also measured the respective rotation

Send offprint requests to: L. Jetsu, e-mail: jetsu@astro.helsinki.fi

* Table 2 is only available in electronic form at the CDS via anonymous ftp to cdarc.u-strasbg.fr (130.79.128.5) or via

http://cdsweb.u-strasbg.fr/cgi-bin/qcat?J/A\&A/383/197

The photometric data are also available at

http://schwab.tsuniv.edu/t3/v511lyr/v511lyr.html velocities $v \sin i=11 \pm 2$ and $11 \pm 3 \mathrm{~km} \mathrm{~s}^{-1}$. Fekel (1997) determined the values of $v \sin i=14.2$ and $13.5 \mathrm{~km} \mathrm{~s}^{-1}$. The most recent estimates are $v \sin i=12 \mathrm{~km} \mathrm{~s}^{-1}$ (G8v) and $10 \mathrm{~km} \mathrm{~s}^{-1}$ (K3v) in Osten \& Saar (1998).

We performed a detailed time series analysis of two years of V511 Lyr photometry. Our modelling results are compiled into Table 1, while the data are published only in electronic form in Table 2.

\section{Observations}

The differential $B V$ photometry of V511 Lyr was made between May 1994 and June 1996 with the VanderbiltTennessee State $0.4 \mathrm{~m}$ Automated Photoelectric Telescope (APT). The comparison and check stars were HDE 337653 $\left(C_{1}\right)$ and HD $179422\left(C_{2}\right)$. Our Table 2 contains seventeen subsets (1st column: SET), the heliocentric Julian dates (2nd column: HJD) and the differential $B V$ magnitudes V511 Lyr minus HDE 337653 (3rd and 4th columns). Note that we analysed sixteen subsets, because SET $=13$ contained only two nights of observations. A suitable subset length was below one month, because the light curves remained stable for about ten rotations (Fig. 1: $P_{\text {phot }} \approx 2$ d 7 ). The time distribution of the data naturally contained gaps without observations, which had to be taken into account when dividing these data into subsets. The selected subsets contain a comparable amount of data, e.g. the number of observing nights has a range between 12 and 19 in 


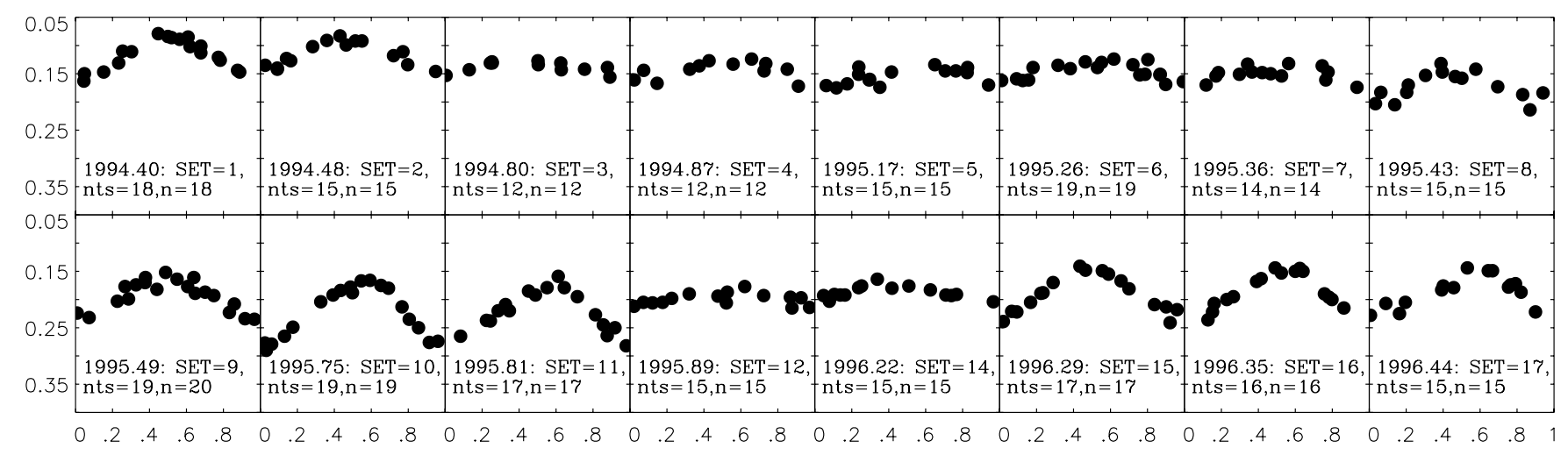

Fig. 1. The differential $V$ magnitudes V511 Lyr minus HDE 337653 with the ephemerides $\mathrm{HJD}_{\min }=t_{\min , 1}+P \times E$ from Table 1 : the epoch (year), the subset number (SET), the number of observing nights (nts) and the number of observations $(n)$.

all analysed subsets (Fig. 1). The photometry was corrected for differential extinction and transformed to the standard Johnson system. Each observation was a mean of three measurements. All observations with a standard deviation greater than 0.01 were automatically rejected. These APT data have already been described by Henry et al. (1995), who studied the SET $=1-4$ photometry. Their external precision estimate, 0.003 , relied on observations of "selected pairs of especially stable stars". This is comparable to our standard deviation of 0.005 between the seasonal $\Delta B_{C_{1}-C_{2}}$ and $\Delta V_{C_{1}-C_{2}}$ values. But the standard deviations within the seasons varied from 0.004 to $0 .{ }^{\mathrm{m}} 008$ in $\Delta V_{C_{1}-C_{2}}$, and from $0 .{ }^{\mathrm{m}} 005$ to 0.009 in $\Delta B_{C_{1}-C_{2}}$. This implies weak variability in $C_{1}$ or $C_{2}$. Considering the spectral-type (Harlan 1969: F5V) and the absence of CaII H\&K emission (Wilson 1966), photometric variability in HD 179422 is improbable. No variability has been observed in this star (e.g. Breger 1969; Fernie 1982) and it has been proved to be a reliable photometric comparison star in studies of the cepheid variable HR 7308 (e.g. Burki et al. 1986). The mean differences in all data were $\Delta B_{C_{1}-C_{2}}=2.605 \pm 0.010$ and $\Delta V_{C_{1}-C_{2}}=2.436 \pm 0.008$. Combining these with $V=6.353$ and $B-V=0.416$ of HD 179422 (Arellano Ferro 1984) gave $V=8.789$ and $B-V=0.585$ for HDE 337653. Except for the spectraltype (Nesterov et al. 1995: F8), the available information about HDE 337653 was limited. Assuming no interstellar extinction, our apparent $V$ magnitude and the Hipparcos satellite 67.6 pc distance estimate (Perryman et al. 1997) indicated a main sequence absolute magnitude (4. 6$)$. Our $B-V$ for HDE 337653 would then fit the G0V spectraltype. In conclusion, if there is real photometric variability in $C_{1}$ or $C_{2}$, it is more probable in the former. But this $\leq 0{ }^{\mathrm{m}} 01$ variability is much weaker than that in V511 Lyr, and does not affect our analysis.

\section{Time series analysis}

The TSPA-method from Jetsu \& Pelt (1999: hereafter Paper I) was applied to search for periodicity in the normalized magnitudes of each subset. Except for the short $\mathrm{SET}=13$, the $B$ and $V$ magnitudes of each subset were normalized (Paper I: Eq. (17)). Our nonlinear second order TSPA model was

$g(t, \bar{\beta})=M+\sum_{k=1}^{2} B_{k} \cos (k 2 \pi f t)+C_{k} \sin (k 2 \pi f t)$.

The free parameters $(\bar{\beta})$ were the mean magnitude $(M)$, the amplitudes $\left(B_{1}, B_{2}, C_{1}, C_{2}\right)$, and the frequency $(f)$. This TSPA analysis gave the primary and secondary minimum epochs $\left(t_{\min , 1}, t_{\min , 2}\right)$ and the photometric rotation period $\left(P=f^{-1}\right)$ in our Table 1 , where the unreliable estimates have been identified with two criteria from Paper I (Sect. 6.3: $R_{\mathrm{I}}$ and $R_{\mathrm{III}}$ ). The former criterion $\left(R_{\mathrm{I}}\right)$ identified the models that did not satisfy Gaussian statistics, while the latter criterion $\left(R_{\mathrm{III}}\right)$ identified the "unreal" $t_{\min , 2}$ estimates. For example, there were six subsets having $n<16$ observations, where the total amplitude of the light curve in $V$ was lower $0{ }^{\mathrm{m}} 05$. The three unreliable $P$ estimates identified with the $R_{\mathrm{I}}$ criterion occurred in these subsets. This confirmed that the modelling of low amplitude light curves with too few observations does not always follow Gaussian statistics. The mean $(M)$ and the total amplitude $(A)$ of the light curves were determined from the original $B V$ magnitudes (Table 1: $M_{B}, M_{V}, A_{B}$ and $A_{V}$ ). This linear modelling with a fixed $f$ was made with the subset ephemeris $\mathrm{HJD}_{\min }=t_{\min , 1}+P \times E$.

The seasonal $V$ light curves revealed that the spot distribution of V511 Lyr sometimes evolved significantly during one month (Fig. 1). The mean $(M)$ reflects the total level of magnetic activity. The amplitude $(A)$ tells how nonuniformly this activity is distributed in longitude. A nearly constant seasonal brightness of V511 Lyr should not be interpreted as absence of magnetic activity, because low $A$ occurred at very different $M$ levels (e.g. Fig. 1: SET $=3$ and 12). The long-term $M_{B}$ and $M_{V}$ changes were smooth (Figs. 2a-c). The effective surface temperature decreased when the star became fainter, i.e. the $M_{B}-M_{V}$ colour index correlated with the mean luminosity (Fig. 2d). The light curve amplitudes $A_{B}$ and $A_{V}$ changed more abruptly than the mean, the most extreme case occurring between SET $=11$ and 12 (Figs. 2e and f). Because the time span of our photometry is only about 
two years, the long-term changes in the mean brightness or the amplitude of the light curve did not give any definite information about the presence of an activity cycle in V511 Lyr. Except for the aforementioned correlation between the colour index and the mean brightness changes, there were no obvious correlations between the different light curve parameters displayed in Fig. 2. However, the time series analysis of the two years of photometry of V511 Lyr did allow us to study two important phenomena already detected in several other chromospherically active stars, namely the differential rotation ( $P$ changes) and the active longitudes $\left(t_{\min , 1}, t_{\min , 2}\right.$ changes).

Henry et al. (1995) discovered the 2.693 periodicity in the SET $=1-2$ photometry, but noted that the 1.59 periodicity was "nearly as strong". The one sidereal day window period $\left(P_{0} \sim 0\right.$ d997) connects these two alternatives and also introduces other spurious periodicities (Paper I: Eq. (16)). The obvious combination for V511 Lyr is $\sim 0.42, \sim 0$. $61, \sim 0.73, \sim 1$. 6 , and $\sim 2$. 7 . Our analysis with the TSPA-method phase residual- and $\chi^{2}$ criteria (Paper I: Sect. 5) confirmed uniquely that $\sim 2$ d 7 is the real periodicity.

If the latitude of the strongest concentrated active region determines the seasonal light curve period, the $P$ changes measure the stellar surface differential rotation. The weighted mean of the reliable $P$ was $P_{\mathrm{w}}=2.710$ with an error of $\Delta P_{\mathrm{w}}=0.017$ (Fig. $2 \mathrm{~g}$ : closed squares). If no significant period changes occur, these reliable period estimates should fit a model of a constant period $P^{\prime}$. The best alternative for such a constant period model is $P^{\prime}=P_{\mathrm{w}}$, because the definition of $P_{\mathrm{w}}$ ensures that no other constant period model can yield a lower $\chi^{2}$ value. We had to reject the hypothesis, "The rotation period of V511 Lyr is constant $\equiv P_{\mathrm{w}}=2$. 710 ", because the reliable $P$ gave $\chi^{2}=98.9$ having a significance $8.9 \times 10^{-16}$. This supported the presence of differential rotation.

The ratio $Z=6 \Delta P_{\mathrm{w}} / P_{\mathrm{w}}$ can be used to measure the statistical $\pm 3 \Delta P_{\mathrm{w}}$ upper limit for the observed period changes. For example, Jetsu et al. (2000) list the values of $Z=7.5 \%, 4.6 \%$ and $3.3 \%$ for V1794 Cyg (G5III-IV), V815 Her (G2v) and V368 Cep (K1V), respectively. They also studied the relation between $Z$ and the differential rotation coefficients $k$ of 15 other stars in Hall \& Busby (1990). Our reliable $P$ estimates from Table 1 gave $Z=3.8 \%$ for $\mathrm{V} 511 \mathrm{Lyr}$. It is also very probable that the full range of the $P$ changes in V511 Lyr has not been observed, because the time span of our photometry is only about two years. Yet, of all 15 stars studied in Hall \& Busby (1990), only the period changes in BY Dra exceeded those observed here in V511 Lyr.

All reliable $P$ estimates were above the three unreliable ones connected to low amplitude light curves (Fig. 2g: open squares). But there was no real correlation between $A$ and $P$, because four other low $A$ light curves had larger and reliable $P$ estimates $(\mathrm{SET}=3,6,12$ and 14$)$. A detailed TSPA analysis example of similar uncertainties encountered with low amplitude light curves was presented in Sect. 6.2 of Paper I. Although no correlation
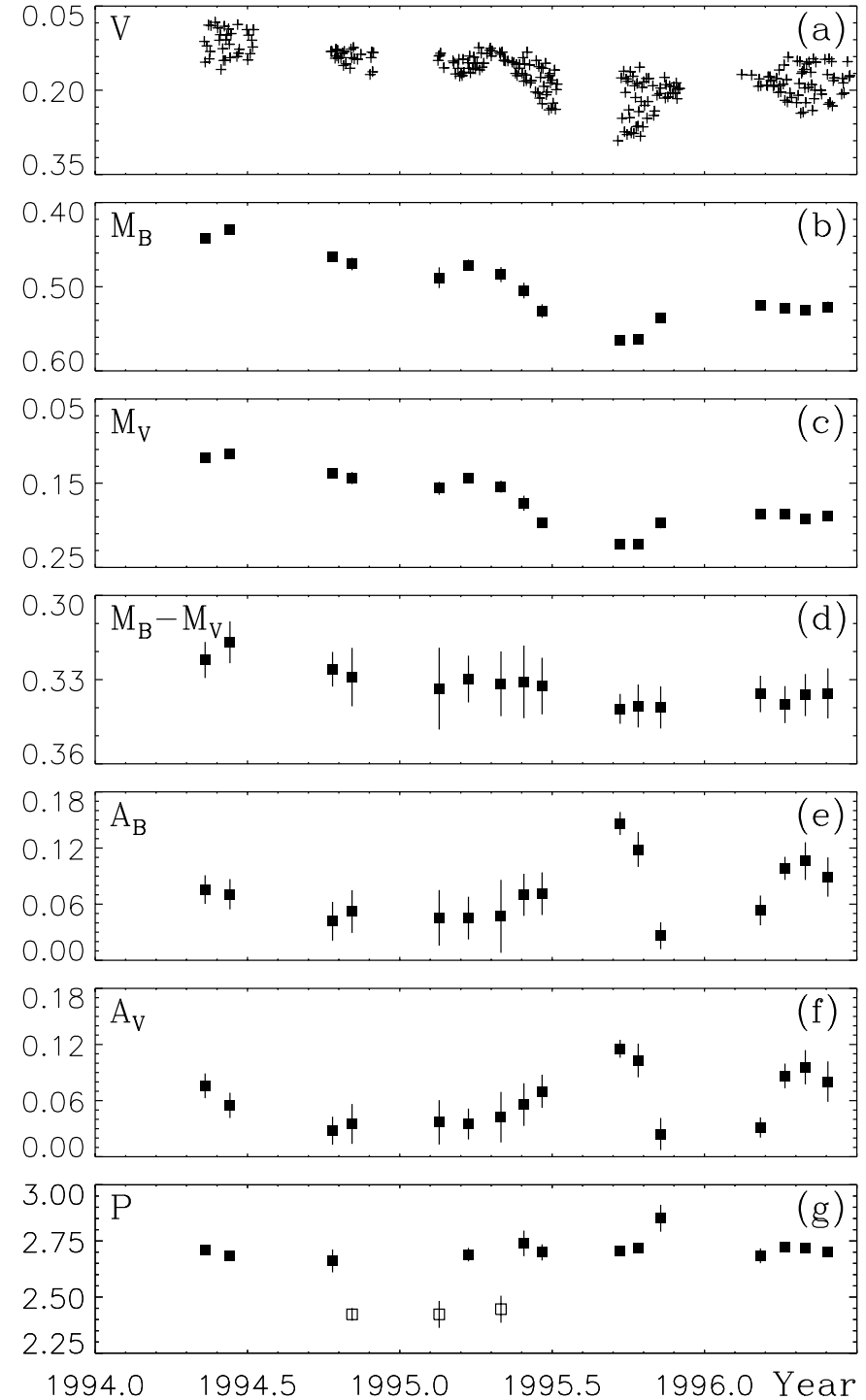

Fig. 2. a) The differential $V$ magnitudes V511 Lyr minus HDE 337653 (Table 2: $n=256$ ). b)-g) The $M_{B}, M_{V}$, $M_{B}-M_{V}, A_{B}, A_{V}$ and $P$ estimates with $\pm 3 \sigma$ error bars. Note that the closed/open squares denote the reliable/unreliable $P$.

between the $A$ and $P$ was found in the light curves of V511 Lyr, such a correlation could be present in some chromospherically active stars. For example, in the case of a strong solar-type differential rotation, where the rotation periods in the equatorial regions are shorter than in the polar regions, the higher $A$ values could be more frequent for the lower $P$ values.

The light curve primary minimum $\left(t_{\min , 1}\right)$ occurs when the strongest active region crosses the central meridian of the visible stellar disk. A secondary minimum $\left(t_{\min , 2}\right)$ represents another weaker active region. We analysed all reliable $t_{\min , 1}$ with the Kuiper-method described in Jetsu \& Pelt (2000, hereafter Paper II). This method was used to test the hypothesis $\left(H_{0}\right)$ : "There is no periodicity in the reliable $t_{\min , 1}$ epochs of V511 Lyr". To cover all $P$ in Table 1 , the periodicities between 2.3 and 3.0 were tested. The best periodicity, $P_{\mathrm{K}}=2.67455 \pm 0$ d.00081, 
Table 1. The photometric rotation period $(P)$, and the primary and secondary minimum epochs $\left(t_{\min , 1}, t_{\min , 2}\right)$, were determined from the normalized magnitudes of each subset (SET). $R_{\mathrm{I}}$ and $R_{\mathrm{III}}$ denote the unreliable estimates. The mean $\left(M_{B}, M_{V}\right)$ and the total amplitude $\left(A_{B}, A_{V}\right)$ were modelled from the original $B V$ differential magnitudes with the subset ephemeris $\mathrm{HJD}_{\min }=t_{\min , 1}+P \times E$. All error estimates are $\pm 1 \sigma$, and have been determined with the bootstrap method (Paper I: Sect. 4)

\begin{tabular}{|c|c|c|c|c|}
\hline \multirow[b]{2}{*}{ SET } & \multicolumn{3}{|c|}{ Normalized magnitudes } & Original $B V$ magnitudes \\
\hline & $P[\mathrm{~d}]$ & $t_{\min , 1}[\mathrm{HJD}]$ & $t_{\min , 2}[\mathrm{HJD}]$ & $A_{B}[\mathrm{mag}] \quad M_{V}[\mathrm{mag}]$ \\
\hline 1 & $2.7119 \pm .0052$ & $2449487.782 \pm .043$ & & $0.4431 \pm .00170 .0756 \pm .00510 .1201 \pm .00130 .0758 \pm .0044$ \\
\hline 2 & $2.6847 \pm .0071$ & $2449517.612 \pm .070$ & & $0.4321 \pm .00190 .0706 \pm .00540 .1154 \pm .00160 .0549 \pm .0045$ \\
\hline 3 & $2.661 \pm .017$ & $2449640.659 \pm .084$ & & $0.4647 \pm .00160 .0417 \pm .00690 .1384 \pm .00130 .0278 \pm .0050$ \\
\hline 4 & $2.4228 \pm .0094 \mathrm{I}$ & $R_{\mathrm{I}} \quad 2449664.01 \pm .16 \quad R$ & & $0.4730 \pm .00250 .0521 \pm .00760 .1439 \pm .00240 .0351 \pm .0071$ \\
\hline 5 & $2.423 \pm .020 \quad I$ & $R_{\mathrm{I}} \quad 2449768.46 \pm .11 R$ & $R_{\mathrm{I}} 2449769.45 \pm .12 R_{\mathrm{I}}$ & $0.4893 \pm .00410 .0454 \pm .00990 .1561 \pm .00260 .0368 \pm .0079$ \\
\hline 6 & $2.689 \pm .010$ & $2449803.522 \pm .061$ & $2449804.73 \pm .14 R_{\mathrm{III}}$ & I $0.4743 \pm .00220 .0451 \pm .00760 .1445 \pm .00170 .0350 \pm .0055$ \\
\hline 7 & $2.446 \pm .020$ & $R_{\mathrm{I}} 2449842.496 \pm .089 R$ & $R_{\mathrm{I}} 2449841.26 \pm .14 R_{\mathrm{I}}$ & $0.4856 \pm .0030 \quad 0.047 \pm .013 \quad 0.1541 \pm .00240 .0423 \pm .0090$ \\
\hline 8 & $2.739 \pm .019$ & $2449870.05 \pm .15$ & & $0.5046 \pm .00310 .0700 \pm .00750 .1738 \pm .00300 .0558 \pm .0076$ \\
\hline 9 & $2.699 \pm .012$ & $2449891.845 \pm .073$ & & $0.5289 \pm .00270 .0712 \pm .00760 .1966 \pm .00200 .0700 \pm .0059$ \\
\hline 10 & $2.7055 \pm .0036$ & $2449985.386 \pm .021$ & & $0.5632 \pm .00130 .1462 \pm .00410 .2228 \pm .00120 .1154 \pm .0032$ \\
\hline 11 & $2.7164 \pm .0056$ & $2450007.006 \pm .031$ & & $0.5623 \pm .00190 .1184 \pm .00620 .2229 \pm .00170 .1029 \pm .0060$ \\
\hline 12 & $2.851 \pm .020$ & $2450033.99 \pm .10$ & $2450035.21 \pm .13 R_{\mathrm{III}}$ & I $0.5375 \pm .00150 .0263 \pm .00480 .1976 \pm .00200 .0243 \pm .0057$ \\
\hline 14 & $2.684 \pm .011$ & $2450153.550 \pm .071$ & & $0.5221 \pm .00180 .0533 \pm .00530 .1870 \pm .00120 .0312 \pm .0036$ \\
\hline 15 & $2.7210 \pm .0047$ & $2450183.023 \pm .037$ & & $0.5255 \pm .00170 .0983 \pm .00410 .1866 \pm .00140 .0864 \pm .0044$ \\
\hline 16 & $2.7192 \pm .0044$ & $2450207.439 \pm .028$ & & $0.5280 \pm .00200 .1060 \pm .00670 .1925 \pm .00150 .0957 \pm .0061$ \\
\hline 17 & $2.7026 \pm .0049$ & $2450234.436 \pm .042$ & & $0.5239 \pm .00210 .0890 \pm .00700 .1890 \pm .00210 .0803 \pm .0072$ \\
\hline
\end{tabular}

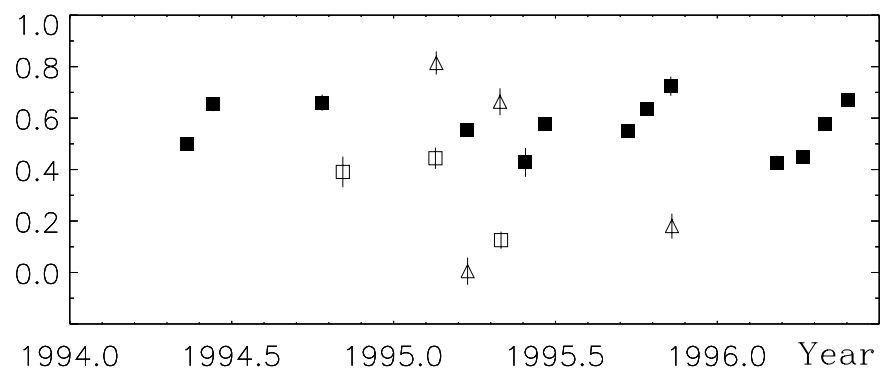

Fig. 3. The primary and secondary minimum phases with the ephemeris $H J D_{\min }=2449486.445+2.67455 \times E$. The closed/open squares denote the reliable/unreliable $\phi_{\min , 1}$. The open triangles show the $\phi_{\min , 2}$ that were all unreliable.

reached the critical level $Q_{\mathrm{K}}=0.0014$ (Paper II: Eq. (A.3)). This critical level $Q_{\mathrm{K}}$ represents the probability that the Kuiper-method test statistic reaches the observed, or an even more extreme, value. Hence the distribution for the phases of the reliable $t_{\min , 1}$ with the best period $P_{\mathrm{K}}$ was extremely improbable. The significance level to reject $H_{0}$ was fixed to $\gamma=0.001$, as in Jetsu (1996), where active longitudes were detected in four RS CVn stars. Although $H_{0}$ could not be rejected in V511 Lyr with this $\gamma$, we emphasize that the critical level of $P_{\mathrm{K}}$ was extremely improbable under $H_{0}$. Furthermore, our Rayleigh-method analysis gave the same best periodicity $P_{\mathrm{R} 1}=2^{\mathrm{d}} \cdot 67426 \pm 0.00084$ with a critical level of $Q_{\mathrm{R} 1}=0.0083$ (Paper II: Eq. (A.4)). The seasonal primary and secondary minimum phases with $P_{\mathrm{K}}$ are displayed in Fig. 3 . The reliable $\phi_{\min , 1}$ concentrated inside an interval of 0.3 in phase. The unreliable $\phi_{\min , 1}$ and $\phi_{\min , 2}$ appeared randomly distributed. These results suggested that the strongest starspot formation on V511 Lyr was concentrated on one active longitude that rotated with a constant period of 2.67455 during the whole time span of our photometry.

Our time series analysis of the photometry has arrived at two apparently contradictory results. The first result was that the reliable seasonal $P$ determined with the TSPA-method exhibited very significant changes. In other words, the hypothesis of a constant rotation period in V511 Lyr had to rejected. This was interpreted as a sign of the presence of differential rotation. The weighted mean of all these seasonal periodicities was $P_{\mathrm{w}}=2 \mathrm{~d} 71$. The second result was that the Kuiper-method analysis of a series of time points, the reliable $t_{\mathrm{min}, 1}$ estimates, indicated the presence of an active longitude rotating with a constant period of $P_{\mathrm{K}}=2^{\mathrm{K}} \cdot 67$. The apparent contradiction is that $P_{\mathrm{w}} \neq P_{\mathrm{K}}$. Furthermore, differential rotation indicates variable rotation periods, but the active longitude indicates a constant rotation period. Nevertheless, similar results have already been obtained in several other chromospherically stars, e.g. in FK Com, V 1794 Cyg, V 815 Her and even in the Sun (Jetsu et al. 1993, 1997, 1999, 2000). In all these cases, the interpretation has been the same. There may exist some long-lived structure(s) below the visible solar or stellar surface that rotate with a constant angular velocity. The formation of the sunor starspots is more frequent close to these structures. When the sun- or starspots are disconnected from these structures, the observed rotation periods depend on their latitudinal location, i.e. on the law of surface differential rotation.

\section{Conclusions}

We studied one signature of magnetic activity in V511 Lyr, the rotational and long-term luminosity changes caused by starspots. The $3.8 \%$ changes in the reliable seasonal 
periodicities $(P)$ determined with the TSPA-method indicated the presence of surface differential rotation. These seasonal periodicity changes were so large that the hypothesis of a constant period had to be rejected. The TSPAmethod also gave the light curve primary and secondary minimum epochs $\left(t_{\min , 1}, t_{\min , 2}\right)$ when the active regions crossed the central meridian of the visible stellar disk. Our Kuiper- and Rayleigh-method analysis of these epochs indicated that the starspot formation in V511 Lyr was concentrated on one active longitude rotating with a constant period of $2 \mathrm{~d} 67455$. These results suggest the coexistence of differential rotation (variable seasonal $P$ ) and active longitudes (constant $P_{\mathrm{K}}$ for all $\left.t_{\mathrm{min}, 1}\right)$. One possible interpretation could be a stable and strong magnetic field structure deeper in the convective zone rotating with a constant period. The variable seasonal periodicities could then represent the latitude of the short-lived starspots emerging to the surface above this structure. The mean brightness $(M)$ changes related to the total starspot area were smooth and correlated with the effective surface temperature. The abrupt changes in light curve amplitudes $(A)$ implied that the longitudinally nonuniform part of the starspot distribution sometimes evolved rapidly, even within one month.

The photometry also provided some new constraints for the binary system V511 Lyr. Without any interstellar extinction, the Hipparcos distance 50.5 pc (Perryman et al. 1997) and the maximum apparent brightness $V=8.87$ implied an absolute magnitude of $M_{V_{1,2}}=5.35$. The most recent estimates for V511 Lyr were $v_{1} \sin i_{1}=$ $12 \mathrm{~km} \mathrm{~s}^{-1}$ (G8v primary) and $v_{2} \sin i_{2}=10 \mathrm{~km} \mathrm{~s}^{-1}$ (K3V secondary) in Osten \& Saar (1998). These particular spectral-types had $M_{V_{1}}=5.6$ with $R_{1}=0.88 R_{\odot}$ and $M_{V_{2}}=6.8$ with $R_{2}=0.73 R_{\odot}$ in Gray (1992). These predicted $M_{V_{1,2}}=5.29$ and a 3.0 flux ratio between the primary and the secondary in $V$. This fits our $M_{V_{1,2}}=5.35$ nicely. Due to the 3.0 flux ratio, the rotation period $P_{\text {phot }}=2.7$ obtained from the light curves most probably represented that of the G8v primary in a binary with $P_{\text {orb }}=2$. 73 . Assuming that the rotation of the less massive K3v secondary is also synchronized, our $P_{\text {phot }}$ and the $v \sin i$ from Osten \& Saar (1998) yielded the projected radii $R_{1} \sin i_{1}=0.64 R_{\odot}$ and $R_{2} \sin i_{2}=0.53 R_{\odot}$. Furthermore, combining these to the radii in Gray (1992) gave consistent inclinations of $i_{1}=47^{\circ}$ and $i_{2}=47^{\circ}$.

Acknowledgements. The research at the Observatory of Helsinki University was performed during spring 2000 by the participants of the lectures "Time Series Analysis in
Astronomy". The work by T.H. was supported by a grant from Wihuri foundation, and by Helsinki University research funding for "Time Series Analysis of Stellar Magnetic Activity" (No. 974/62/98). Astronomy with automated telescopes at Tennessee State University is supported through NASA grants NCC5-96 and NCC5-228, which funds TSU's Center for Automated Space Science, and NSF grant HRD-9706268, which funds TSU's Center for Systems Science Research. We have made use of the Simbad-database operated at CDS, Strasbourg, France. We wish thank the anonymous referee for valuable comments on our manuscript.

\section{References}

Arellano Ferro, A. 1984, MNRAS, 209, 481

Bidelman, W. P. 1983, AJ, 88, 1182

Breger, M. 1969, ApJS, 19, 79

Burki, G., Schmidt, E. G., Arellano Ferro, A., et al. 1986, A\&A, 168, 139

Fekel, F. C. 1997, PASP, 109, 514

Fernie, J. D. 1982, PASP, 94, 537

Gray, D. F. 1992, The observation and analysis of stellar photospheres, 2nd Edition (Cambridge Univ. Press, Cambridge)

Hall, D. S., \& Busby, M. R. 1990, in Active Close Binaries, ed. C. Ibanog̃lu (Kluwer, Dordrecht), 377

Harlan, E. A. 1969, AJ, 74, 916

Henry, G. W., Fekel, F. C., \& Hall, D. S. 1995, AJ, 110, 2926

Jeffries, R. D., Bertram, D., \& Spurgeon, B. R. 1995, MNRAS, 276, 397

Jetsu, L. 1996, A\&A, 314, 153

Jetsu, L., \& Pelt, J. 1999, A\&AS, 139, 629, Paper I

Jetsu, L., \& Pelt, J. 2000, A\&A, 353, 409, Paper II

Jetsu, L., Pelt, J., \& Tuominen, I. 1993, A\&A, 278, 449

Jetsu, L., Pohjolainen, S., Pelt, J., \& Tuominen, I. 1997, A\&A, 318,293

Jetsu, L., Pelt, J., \& Tuominen, I. 1999, A\&A, 351, 212

Jetsu, L., Hackman, T., Hall, D. S., et al. 2000, A\&A, 362, 223

Lampton, M., Lieu, R., Schmitt, J. H. M. M., et al. 1997, ApJS, 108, 545

Mason, K. O., Hassall, B. J. M., Bromage, G. E., et al. 1995, MNRAS, 274, 1194

Mulliss, C. L., \& Bopp, B. W. 1994, PASP, 106, 822

Nesterov, V. V., Kuzmin, A. V., Ashimbaeva, N. T., et al. 1995, A\&AS, 110, 367

Osten, R. A., \& Saar, S. H. 1998, MNRAS, 295, 257

Perryman, M. A. C., Lindegren, L., Kovalevsky, J., et al. 1997, A\&A, 323, L49

Pounds, K. A., Allan, D. J., Barber, C., et al. 1993, MNRAS, 260, 77

Pye, J. P., McGale, P. A., Allan, D. J., et al. 1995, MNRAS, 274, 1165

Wilson, O. C. 1966, ApJ, 144, 695 